\title{
Proton form factors in space-like and time-like regions
}

\author{
Simone Pacetti* \\ Dipartimento di Fisica e Geologia dell'Università degli Studi di Perugia and INFN Sezione di \\ Perugia, 06123, Italy \\ E-mail: simone.pacetti@pg.infn.it
}

\section{Rinaldo Baldini Ferroli}

INFN, Laboratori Nazionali di Frascati, 00044 Frascati, Italy

E-mail: rinaldo.baldinielnf.infn.it

\section{Egle Tomasi-Gustafsson}

CEA, IRFU, SPhN, Saclay, 91191 Gif-sur-Yvette Cedex, France

E-mail: egle.tomasi@cea.fr

Electromagnetic proton form factors are reviewed by describing their fundamental properties and also by focusing on the still obscure issues. All available data are presented. A typical and successful phenomenological description is treated by highlighting some of its general aspects.

XXII International Baldin Seminar on High Energy Physics Problems,

15-20 September 2014

JINR, Dubna, Russia

${ }^{*}$ Speaker. 


\section{The Facts}

Form factors describe the non point-like nature, with respect to a given interaction, of a physical system, i.e., the fact that, if sounded out with the probe of that specific interaction, it behaves as an extended and continuous spatial distribution of scattering points. In case of electromagnetic interaction, and hence using elementary charged particles as probes (e.g. electrons), the spatial charge distribution is investigated. By following such a semi-classical point of view, the electromagnetic form factor $(\mathrm{FF})$ of a particle, is defined as

$$
F(\vec{q})=\int d^{3} \vec{x} e^{i \vec{x} \cdot \vec{q}} \rho(\vec{x})
$$

it is the Fourier transform of the electric charge distribution $\rho(\vec{x})$, so it depends on the threemomentum $\vec{q}$ transferred by the point-like probe to the extended target, where the concept of spacial extension depends on the interaction. At zero momentum transfer the FF equals the total electric charge, usually defined in units of the positron charge, so that $F(0)$ is a dimensionless positive or negative integer number. For instance in the case of the proton: $F(0)=1$.

In quantum field theory (QFT) FFs come on stage when vertices involving elementary interactioncarriers together with non-elementary particles are considered. Form factors have to be introduced to parametrize the proton electromagnetic four-current, $J^{\mu}\left(q=k^{\prime}-k\right)$, i.e., the amplitude of the process $p(k) \rightarrow \gamma^{*}(q)+p\left(k^{\prime}\right)$, where $k$ and $k^{\prime}$ are the initial and final four-momenta. In particular, such a current is defined, in terms of proton Dirac spinors and a combination, $\Gamma^{\mu}$, of gamma matrices and their products, as

$$
J^{\mu}(q)=\bar{u}\left(k^{\prime}\right) \Gamma^{\mu} u(k), \quad \Gamma^{\mu}=\gamma^{\mu} F_{1}\left(q^{2}\right)+\frac{i \sigma_{\mu v} q^{v}}{2 M} F_{2}\left(q^{2}\right),
$$

where $M$ is the proton mass. The coefficients that weight the vector $\left(\gamma^{\mu}\right)$ and tensor $\left(\sigma_{\mu v}\right)$ part of $\Gamma^{\mu}$, which, as a consequence of gauge and Lorentz invariance, are scalar functions, represent the Dirac and Pauli FFs, respectively. With respect to the semi-classical FF definition, given in eq. (1.1), two new features arise considering FFs in QFT: even more than one FF could be needed for a single hadron depending on its spin ( $2 S+1$ for a hadron of spin $S$ ) and, by invoking crossing symmetry, the same FFs describe both the scattering and production processes, i.e., the vertices: $p(k) \rightarrow \gamma^{*}(q)+p\left(k^{\prime}\right)$ and $\gamma^{*}(q) \rightarrow p(k)+\bar{p}\left(k^{\prime}\right)$, for space-like (SL) and time-like (TL) fourmomenta $q$. The decomposition of $\Gamma^{\mu}$ in terms of the Dirac and Pauli FFs is not unique, equivalent pairs of FFs can be defined. They correspond to representations of $\Gamma^{\mu}$ with respect to different basis in the gamma matrix vector space. A useful choice, besides that of eq. (1.2), is represented by the so-called Sachs FFs, electric and magnetic, defined as

$$
G_{E}\left(q^{2}\right)=F_{1}\left(q^{2}\right)+\frac{q^{2}}{4 M^{2}} F_{2}\left(q^{2}\right), \quad G_{M}\left(q^{2}\right)=F_{1}\left(q^{2}\right)+F_{2}\left(q^{2}\right) .
$$

In the kinematical region where there is no energy exchanged, i.e., for $q=(0, \vec{q})$, they represent the Fourier transforms of the electric charge and magnetization spatial distributions of the proton. As a consequence, the normalization at $q^{2}=0$ is given in terms of the total electric charge, $Q=1$, and magnetic moment, $\mu=\mu_{p}=2.793$, in units of the positron electric charge and the Bohr magneton 
respectively, so that

$$
\left\{\begin{array}{l}
G_{E}(0)=1 \\
G_{M}(0)=\mu_{p}
\end{array}, \quad\left\{\begin{array}{l}
F_{1}(0)=1 \\
F_{2}(0)=\mu_{p}-1
\end{array} .\right.\right.
$$

Further information about proton FFs, mainly concerning their behavior as functions of $q^{2}$, can be derived from first-principles considerations.

Analyticity. There are two different approaches to infer the analyticity of FFs. The first relies on the micro-causality requirement, while the second is based on QFT, that is, in a pure perturbative procedure. In both cases, FFs are proven to be analytic functions in the $q^{2}$ complex plane with the cut $\left(q_{\mathrm{th}}^{2}, \infty\right)$, along the positive real axis, see Fig. 1 .

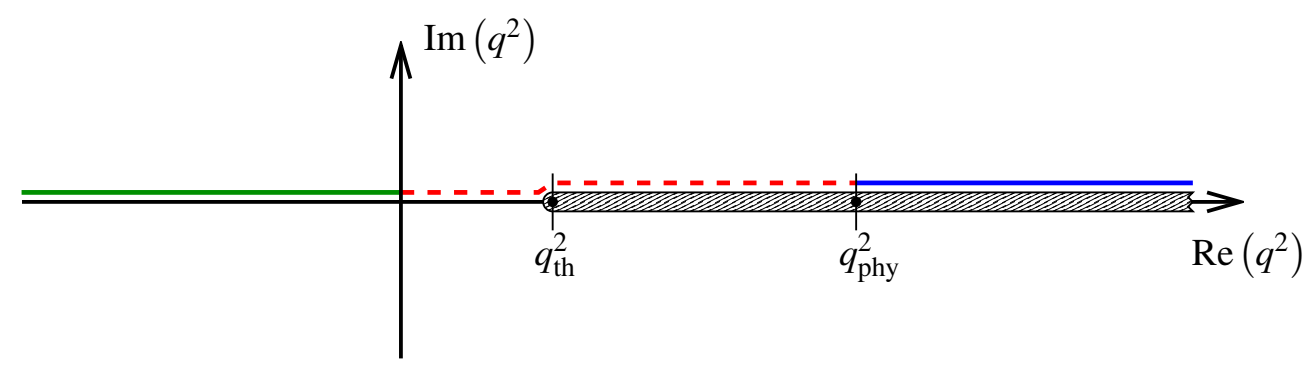

Figure 1: The $q^{2}$ complex plane. Colored solid lines over the real axis indicate the kinematical regions, $\mathrm{SL}$ and TL, in green and blue respectively, where FFs are experimentally accessible. The red dashed line represents the "unphysical region" (see text).

The value $q_{\mathrm{th}}^{2}$ represents the theoretical threshold and corresponds to the mass squared of the lightest hadronic channel, that has the same quantum numbers of the considered final state, i.e., the two-pion channel, so that $q_{\mathrm{th}}^{2}=\left(2 M_{\pi}\right)^{2}$, being $M_{\pi}$ the pion mass. The discontinuity cut is a consequence of unitarity and it can be quantitatively described in terms of the optical theorem. It determines a crucial properties of FFs: their complexity for values of $q^{2}$ above the threshold $q_{\mathrm{th}}^{2}$, while for real $q^{2} \leq q_{\mathrm{th}}^{2}$ FFs are real. This is due to the hermiticity of the current operator.

Threshold. The production or physical threshold, in the TL region, is $q_{\text {phy }}^{2}=(2 M)^{2}$ and corresponds to the squared four-momentum at which the proton and the antiproton are produced at rest in their center of mass system (CM). Apart from the experimental relevance of $q_{\mathrm{phy}}^{2}$, indeed is the lower squared TL four-momentum at which FF data are available ${ }^{1}$, such a threshold has no special meaning for FFs. In fact, they should maintain a smooth behavior passing from $q^{2}<q_{\text {phy }}^{2}$ to $q^{2}>q_{\text {phy }}^{2}$. Moreover, assuming regular FFs and hence no singular behavior for $F_{1}\left(q^{2}\right)$ and $F_{2}\left(q^{2}\right)$, Sachs FFs must coincide at $q_{\text {phy }}^{2}$. On the other hand, experimental evidences of the inequality $G_{E}\left(q_{\text {phy }}^{2}\right) \neq G_{M}\left(q_{\text {phy }}^{2}\right)$ would imply that the Dirac and Pauli FFs have a simple pole at $q_{\text {phy }}^{2}$.

Asymptotia. At high- $\left|q^{2}\right|$, perturbative QCD (pQCD) as well as dimensional counting rule [3], can be used to predict the SL asymptotic trend of FFs, namely their behavior as $q^{2} \rightarrow-\infty$. In particular

\footnotetext{
${ }^{1}$ Studies to experimentally implement an old idea to overcome such a limitation, are in progress [2].
} 
it is found

$$
\mathscr{F}\left(q^{2}\right)=O\left[\left(q^{2}\right)^{-2}\right], \quad q^{2} \rightarrow-\infty,
$$

with $\mathscr{F}\left(q^{2}\right)=G_{E}\left(q^{2}\right), G_{M}\left(q^{2}\right)$. This means that, apart from QCD corrections due to the running of the strong coupling constant, both Sachs FFs vanish with the same power, -2 , of $q^{2}$. The same behavior is also expected in the TL limit $q^{2} \rightarrow+\infty$. Indeed, the analyticity and the boundedness in the $q^{2}$ complex plane, deprived of the half-line $\left(q_{\mathrm{th}}^{2}, \infty\right)$, imply, as a consequence of the Phragmèn-Lindelöf theorem [4] $]^{2}$, that FFs have the same limit as $q^{2}$ diverges, not only along the negative (SL $q$ ) and positive (TL $q$ ) real axis, but also in any other direction, i.e.,

$$
\lim _{q^{2} \rightarrow \infty} \mathscr{F}\left(q^{2} e^{i \theta}\right)=\lim _{q^{2} \rightarrow \infty} \mathscr{F}\left(q^{2} e^{i \pi}\right), \quad \forall \theta \in(0,2 \pi),
$$

where the phase $\theta=\pi$ identifies the negative real axis and hence the SL region. As an interesting consequence, FFs in the TL region, as $q^{2} \rightarrow \infty$, become real and equal to the symmetric SL value, i.e., their imaginary part vanishes faster than the real one. This also means that the phase $\phi\left(q^{2}\right)$, defined through the relation

$$
\mathscr{F}\left(q^{2}\right)=\left|\mathscr{F}\left(q^{2}\right)\right| e^{i \phi\left(q^{2}\right)},
$$

goes to an integer multiple of $\pi$ radians, as $q^{2} \rightarrow \infty$.

\section{The data}

Experimental values of FFs are drawn from several sources. Different experiments investigating processes which are connected by crossing symmetry, are needed to cover different kinematical regions. Moreover, single processes are studied by exploiting more techniques to access as complete as possible information on FFs.

Space-like The SL region has been the first explored, since 1960, by the pioneering experiments of
region. Hofstadter [5], based on the measurement of the cross section for unpolarized electron-proton elastic scattering. In Born approximation, i.e., by considering only one-photon exchange, the unpolarized differential cross section for the scattering process $e^{-}+p \rightarrow e^{-}+p$ in the proton rest frame, that is the laboratory frame (LAB), reads

$$
\frac{d \sigma_{e p}}{d \Omega}=\frac{\alpha^{2} \varepsilon_{2} \cos ^{2}\left(\frac{\theta_{e}}{2}\right)}{4 \varepsilon_{1}^{3} \sin ^{4}\left(\frac{\theta_{e}}{2}\right)}\left\{G_{E}^{2}\left(q^{2}\right)-\tau\left[1+2(1-\tau) \tan ^{2}\left(\frac{\theta_{e}}{2}\right)\right] G_{M}^{2}\left(q^{2}\right)\right\} \frac{\tau}{1-\tau}, \quad \tau=\frac{q^{2}}{4 M^{2}},
$$

where: $\alpha$ is the fine-structure constant, $\varepsilon_{1(2)}$ is the energy of the incoming (outgoing) electron, $\theta_{e}$ is the electron scattering angle. For this process the four-momentum transfer is SL.

\footnotetext{
${ }^{2}$ Actually, the analyticity and the boundedness of FFs in the upper-half $q^{2}$ complex plane $\left(\operatorname{Im}\left(q^{2}\right)>0\right)$ is a sufficient condition for the applicability of the Phragmèn-Lindelöf theorem, however in our case FFs are real for real $q^{2} \leq q_{\mathrm{th}}^{2}$ and hence respect the Schwarz reflection principle: $\mathscr{F}^{*}\left(q^{2}\right)=\mathscr{F}\left(q^{2 *}\right)$, which extend analyticity and boundedness from the upper to the lower half-plane.
} 
Indeed, in terms of the initial, $p_{i}$, and final, $p_{f}$, proton four-momenta, we have $q=p_{f}-p_{i}$ so that, using the LAB expressions $p_{i}=(M, 0)$ and $p_{f}=\left(E_{f}, \vec{p}_{f}\right)$, it is $q^{2}=2 M\left(M-E_{f}\right)<0$. Values of $G_{E}^{2}\left(q^{2}\right)$ and $G_{M}^{2}\left(q^{2}\right)$, that are real in this kinematical region, are extracted by means of the so-called Rosenbluth technique [6], i.e., by measuring the cross section at different angles $\theta_{e}$ and fixed $q^{2}$. Since the electric and magnetic FFs contribute to the cross section with different, $q^{2}$-dependent weights, lower order radiative corrections (RC), implemented as an overall factor, play a crucial role in the determination of $G_{E}\left(q^{2}\right)$ and $G_{M}\left(q^{2}\right)$ (for a detailed discussion on RC see e.g. Ref. [1]).

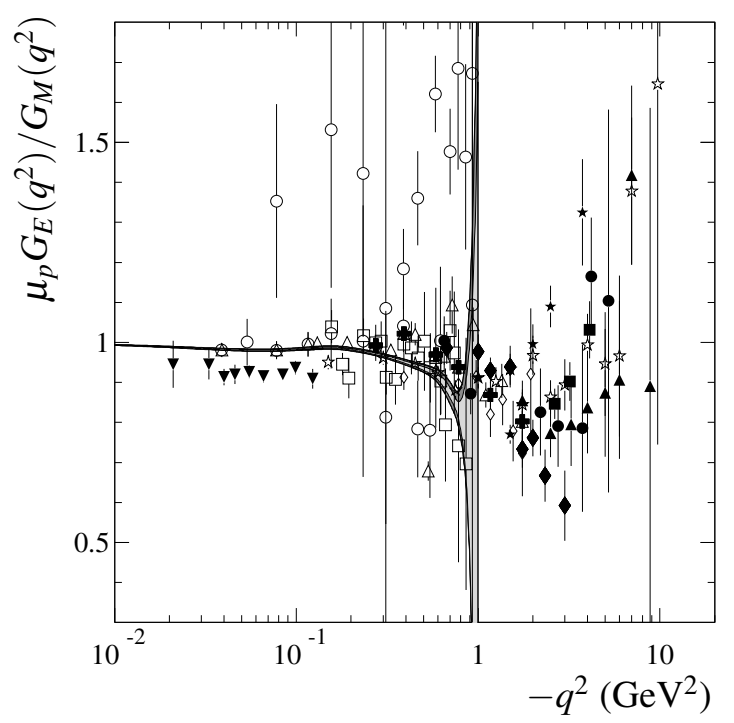

Figure 2: World data on $\mu_{p} G_{E}\left(q^{2}\right) / G_{M}\left(q^{2}\right)$ as a function of $q^{2}$ from unpolarized $e^{-} p$-elastic scattering. Data and symbols are those of Fig. 8 in Ref. [1].

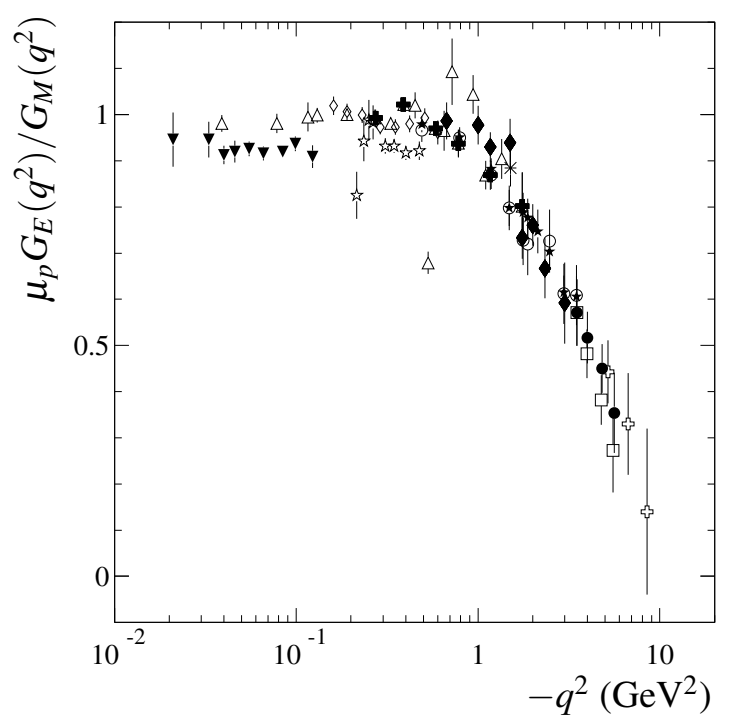

Figure 3: Ratio $\mu_{p} G_{E}\left(q^{2}\right) / G_{M}\left(q^{2}\right)$ from the polarization technique, compared with a set of selected unpolarized data (empty triangles, solid diamonds, solid crosses, solid down-triangles). Data and symbols are those of Fig. 9 in Ref. [1].

Figure 2 show all the available data, obtained by using the Rosenbluth technique, on the ratio electric to magnetic proton FF normalized to the proton magnetic moment $\mu_{p}$. These data strongly support the so-called scaling, i.e., the coincidence of the normalized proton Sachs FFs, at least in the low- $\left|q^{2}\right|$ region, i.e., $G_{E}\left(q^{2}\right)=G_{M}\left(q^{2}\right) / \mu_{p}$.

Following the original idea of Akhiezer and Rekalo [7], that in 1968 showed how double spin polarization observables in elastic electron-proton could be used to extract data directly on the ratio, for the first time in a series of experiments at the Jefferson Laboratory (JLab), since 1999 , the ratio $G_{E}\left(q^{2}\right) / G_{M}\left(q^{2}\right)$ has been obtained by measuring the polarization transferred to the scattered protons by the longitudinally polarized electrons [8]. In more detail, in the LAB, the ratio between the transverse, $P_{T}\left(q^{2}\right)$, and the longitudinal, $P_{L}\left(q^{2}\right)$, component of the polarization vector of the scattered proton is

$$
\frac{P_{T}\left(q^{2}\right)}{P_{L}\left(q^{2}\right)}=-\frac{2 M \cot \left(\theta_{e} / 2\right)}{\varepsilon_{1}+\varepsilon_{2}} \frac{G_{E}\left(q^{2}\right)}{G_{M}\left(q^{2}\right)} .
$$

The data on $G_{E}\left(q^{2}\right) / G_{M}\left(q^{2}\right)$ obtained with this procedure are expected to be very precise, because, at first order, the beam helicity as well as the analyzing power of the proton po- 
larimeter cancel out, by reducing the systematic error and by improving the sensitivity to small contributions of $G_{E}\left(q^{2}\right)$.

The expectation about the precision was not disappointed, however, the most surprising feature of these new data was the trend they showed, a monotone decreasing behavior, almost linear in $-q^{2}$, as shown in Fig. 3. Such a tendency seems to herald the vanishing of the ratio and hence, due to the analyticity of FFs, a zero for $G_{E}\left(q^{2}\right)$ at $-q^{2} \simeq 10 \mathrm{GeV}^{2}$.

Time-like region.

To extract TL values of FFs the crossed reactions of the electron-proton scattering, i.e., the annihilations $p+\bar{p} \leftrightarrow e^{+}+e^{-}$, have to be studied. Their unpolarized differential cross sections, in CM and in Born approximation, apart from the phase-space factor, have the same dependence on FFs [9]

$$
\frac{d \sigma_{p \bar{p}, n}}{d \Omega}=\frac{\alpha^{2}}{4 q^{2}} \beta^{2 n-1}\left[\left(1+\cos ^{2}(\theta)\right)\left|G_{M}\left(q^{2}\right)\right|^{2}+\frac{1}{\tau} \sin ^{2}(\theta)\left|G_{E}\left(q^{2}\right)\right|^{2}\right],
$$

where $\theta$ is the scattering angle, $\beta$ is the velocity of the proton and the power $(2 n-1)$ defines the reaction, i.e.,

$$
\beta=\sqrt{1-\frac{4 M^{2}}{q^{2}}}, \quad\left\{\begin{array}{l}
n=0 \text { for: } e^{+}+e^{-} \rightarrow p+\bar{p} \\
n=1 \text { for: } p+\bar{p} \rightarrow e^{+}+e^{-}
\end{array} .\right.
$$

In these processes the four-momentum transferred $q$, which is the sum of the four-momenta of the proton and antiproton, $p_{1}$ and $p_{2}$, is TL. Indeed, by using the CM expressions: $p_{1,2}=(E, \pm \vec{p})$, we have: $q^{2}=\left(p_{1}+p_{2}\right)^{2}=4 E^{2} \geq 4 M^{2}=q_{\text {phy }}^{2}$. Moreover, such a differential cross section depends only on the moduli of FFs, which are complex (have non vanishing imaginary parts) for real $q^{2}$ lying above the theoretical threshold $q_{\mathrm{th}}^{2}$. It follows that, even in an ideal experimental framework, by studying unpolarized observables of the annihilation processes $p+\bar{p} \leftrightarrow e^{+}+e^{-}$, only the moduli of FFs for $q^{2} \geq q_{\text {phy }}^{2}$ are measurable. In other words, FFs are completely unobservable for $q^{2} \in\left[0, q_{\text {phy }}^{2}\right]^{3}$, the so-called "unphysical region", see fig. 1, while their complex phases remain unknown everywhere in the $q^{2}$ complex plane. The individual determination of $\left|G_{E}\left(q^{2}\right)\right|$ and $\left|G_{M}\left(q^{2}\right)\right|$ has been attempted by only two experimental Collaborations, in 1993 at the LEAR antiproton ring at CERN [10] and, in 2005 [11] and 2013 [12], by BABAR at the $e^{+} e^{-}$-collider PEP-II at SLAC. The BABAR data has been obtained by using the "initial state radiation" technique (ISR), that mimics the same observables of typical $e^{+} e^{-}$experiment, with CM energy scan, at fixed energy machines, i.e., at the flavor factories. Data on the $e^{+}+e^{-} \rightarrow H$ cross section, where $H$ stands for a generic final state (in our case $H=p+\bar{p}$ ), as a function of the $e^{+} e^{-}-\mathrm{CM}$ energy, are extracted from the differential cross section of the three-body process $e^{+} e^{-} \rightarrow p+\bar{p}+\gamma$, with the photon emitted by one of the initial leptons (see Refs. [11, 12] for more details). Figure 4 shows the two sets of data that, in different but superimposed interval of $q^{2}$, are not compatible. While BABAR data (open circles in Fig. 4) demonstrate clearly the deviation from the unity of the ratio, the points from LEAR (open squares in Fig. 4) still agree with the identity $\left|G_{E}\left(q^{2}\right)\right|=\left|G_{M}\left(q^{2}\right)\right|$, which is true only at $q^{2}=q_{\text {phy }}^{2}$ (see Sec. 1).

\footnotetext{
${ }^{3}$ See footnote 1 .
} 


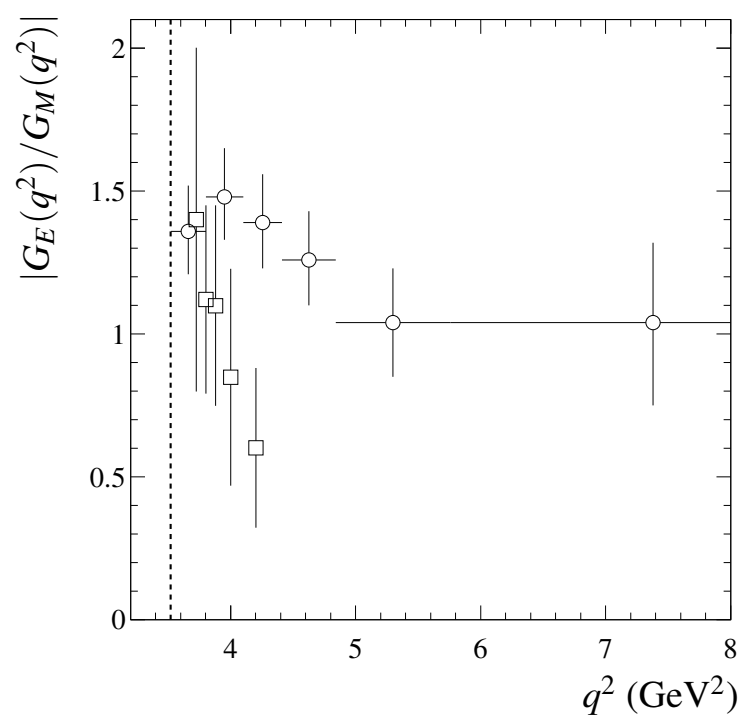

Figure 4: Modulus of the ratio $G_{M}\left(q^{2}\right) / G_{M}\left(q^{2}\right)$ in the TL region. The data are from: Ref. [12] (open circles) and Ref. [10] (open squares). The vertical dashed line indicates the physical threshold $q_{\text {phy }}^{2}=4 M^{2}$.

Time-like data on FFs are often given in terms of an effective FF, $G_{\text {eff }}\left(q^{2}\right)$, corresponding to the assumption $\left|G_{E}\left(q^{2}\right)\right|=\left|G_{M}\left(q^{2}\right)\right| \equiv G_{\text {eff }}\left(q^{2}\right)$, under which, the total cross section becomes

$\sigma_{p \bar{p}, n}=\frac{2 \pi \alpha^{2}}{3 q^{2}} \frac{\beta^{2 n-1}}{\tau}\left[2 \tau\left|G_{M}\left(q^{2}\right)\right|^{2}+\left|G_{E}\left(q^{2}\right)\right|^{2}\right] \rightarrow \sigma_{p \bar{p}, n}=\frac{2 \pi \alpha^{2}}{3 q^{2}} \frac{\beta^{2 n-1}}{\tau}[2 \tau+1] G_{\mathrm{eff}}^{2}\left(q^{2}\right)$,

and hence the definition of $G_{\text {eff }}$ in terms of moduli of Sachs FFs reads

$$
G_{\mathrm{eff}}\left(q^{2}\right)=\sqrt{\frac{2 \tau\left|G_{M}\left(q^{2}\right)\right|^{2}+\left|G_{E}\left(q^{2}\right)\right|^{2}}{2 \tau+1}} .
$$

Figure 5 shows, in two panels to highlight the low and high- $q^{2}$ region, the world data set on $G_{\text {eff }}\left(q^{2}\right)$. The main features of these data are: the threshold behavior, indeed $G_{\text {eff }}\left(q^{2}\right)$ has a very steep negative slope, which is actually due to a total cross section $\sigma_{p \bar{p}, 0}$ almost flat for about $0.8 \mathrm{GeV}^{2}$ above $q_{\text {phy }}^{2}$; the presence of several structures; the general trend follows the expected power law, i.e., $G_{\text {eff }}\left(q^{2}\right) \propto\left(q^{2}\right)^{-2}$, as $q^{2} \rightarrow \infty$.

\section{Long standing and recent issues}

It is undeniable that the results obtained at JLab by the GEP collaboration [8], showing an astonishing behavior of the SL ratio $\mu_{p} G_{E}\left(q^{2}\right) / G_{M}\left(q^{2}\right)$, that seems to vanish instead of remaining constant and equal to one, injected new vital lymph in the nucleon FF communities, both the experimental and the theoretical one. However, such a new interest has also brought back to light issues that are still open. 

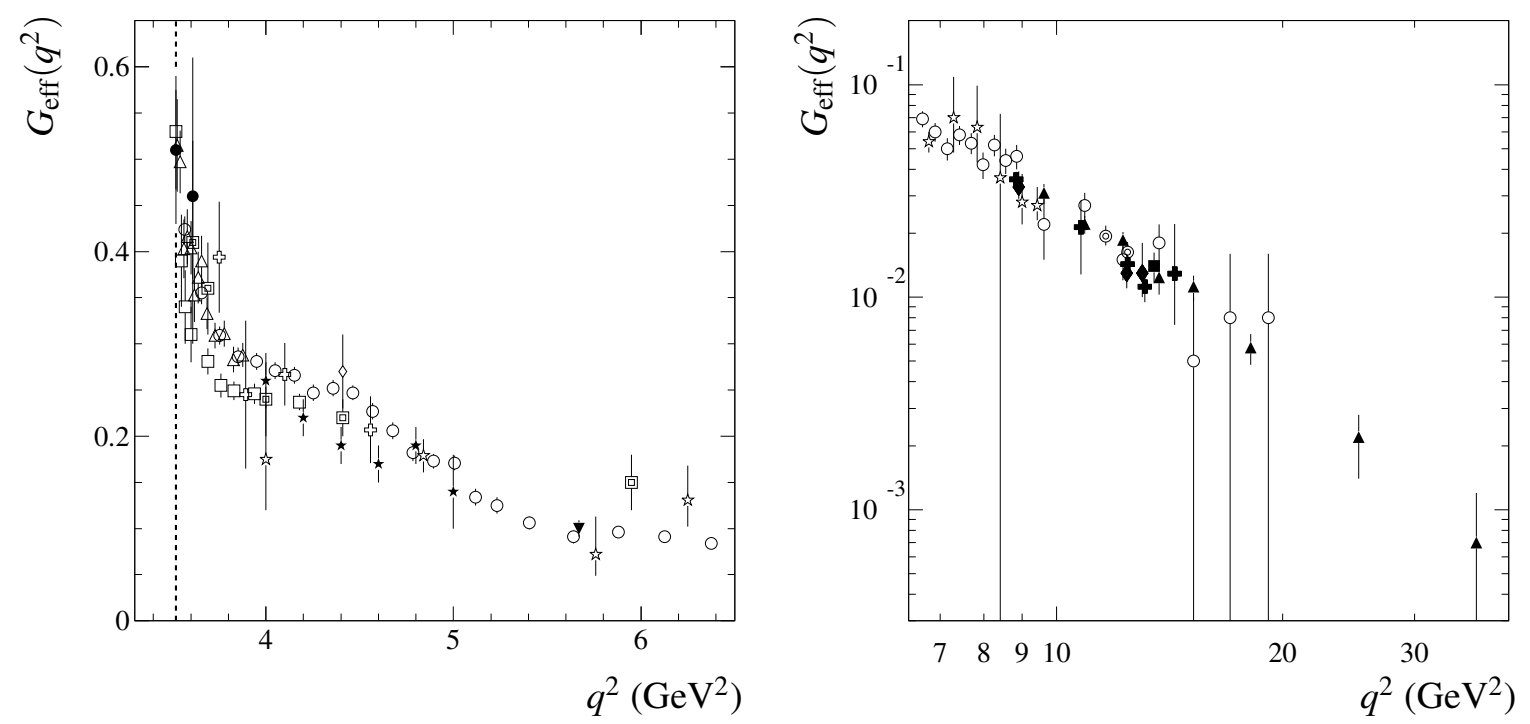

Figure 5: World data of the effective proton FF, $G_{\text {eff }}\left(q^{2}\right)$, in the low (left panel) and high (right panel) momentum transfer regions. The vertical dashed line in the left panel indicates the physical threshold $q_{\text {phy }}^{2}$. Data and symbols are those of Fig. 10 in Ref. [1].

- The most important one is just the "discovery" done at JLab, that has two implications. The first concerns the physical interpretation of this result, i.e., the meaning of a possible zero for the electric proton FF, $G_{E}\left(q^{2}\right)$.

The second implication deals with the discrepancy between the two procedures, polarization and Rosenbluth, that has to be understood. Since in using the polarization technique, systematic errors as well as radiative corrections are better under control, the results obtained with this procedure appear more reliable and are commonly considered the right ones.

For the same reasons, a "not sufficiently accurate" implementation of radiative corrections appears as the most likely explanation for the polarization-Rosenbluth discrepancy.

In this line of thought, two different theoretical approaches emerged. Some authors identify in an underestimate of the two-photon exchange contribution, that for some mechanism could be larger than a usual order- $\alpha$ correction, the main cause of the discrepancy [13]. For some others, instead, polarization and Rosenbluth results can be reconciled by considering higher-order "standard" radiative corrections [14].

- The root-mean-square charge radius of the proton is defined in terms of the logarithmic derivative of the electric FF at $q^{2}=0$ as

$$
r_{p}=\sqrt{\left\langle r_{p}^{2}\right\rangle}=\sqrt{\left.6 \frac{d \ln \left[G_{E}\left(q^{2}\right)\right]}{d q^{2}}\right|_{q^{2}=0}}=\sqrt{\left.\frac{6}{G_{E}(0)} \frac{d G_{E}\left(q^{2}\right)}{d q^{2}}\right|_{q^{2}=0}},
$$

so that it does not depend on the normalization at $q^{2}=0$. Its value can be determined from the SL data on $G_{E}\left(q^{2}\right)$, however the precision achieved is heavily limited by both, the experimental difficulties in reaching four-momenta transferred sufficiently close to zero and the theoretical problems concerning the corresponding radiative corrections. Moreover, recent measurements of $r_{p}$ performed by using the Lamb shift in muonic hydrogen [15] gave a value 
of the radius lower than the previous measurements (from $G_{E}\left(q^{2}\right)$ and using Lamb shift in electronic hydrogen) and so precise to have a discrepancy of eight standard deviations. A better knowledge of $G_{E}\left(q^{2}\right)$ in the $\mathrm{SL}$ and $\mathrm{TL}^{4}$ region is fundamental to improve the precision in the extraction of $r_{p}$ and hence to achieve a deeper understanding of this disagreement.

- The TL threshold region turns out to be very intriguing. Indeed, to account for the electromagnetic interaction between the proton and antiproton when they are produced or annihilated very close to threshold, i.e., when the velocities in their CM are vanishing, an overall correction factor has to be considered in the cross section expression. It is called Coulomb factor and has the form: $\mathscr{C}=\left|\psi_{0}(0)\right|^{2}$, where $\psi_{L}(r)$ is the wave function solution of the Schrödinger equation with a Coulomb potential and orbital angular momentum $L$ [16]. Its non-relativistic nature is justified by the fact that it becomes effective (more than few \%) only in that limit, i.e., when $\beta \rightarrow 0$, being $\beta$ the proton and antiproton velocity in the $p \bar{p} \mathrm{CM}$.

Since $\mathscr{C}$ goes like $\beta^{-1}$, in case of $e^{+}+e^{-} \rightarrow p+\bar{p}$, the Coulomb factor plays the specific role of compensating the natural "closure" of the phase-space, occurring at $\beta=0$, so that it induces a non vanishing cross section at the threshold, $q_{\text {phy }}^{2}$ [17]. Moreover, such a threshold cross section depends only on the moduli of FFs, that at $q^{2}=q_{\text {phy }}^{2}$ coincide. Ultimately, by indicating with $\left|G\left(q_{\text {phy }}^{2}\right)\right|$ the common value of $\left|G_{M}\left(q^{2}\right)\right|$ and $\left|G_{M}\left(q^{2}\right)\right|$ at the physical threshold, we have

$$
\sigma_{p \bar{p}, 0}\left(q_{\text {phy }}^{2}\right)=(0.85 \mathrm{nb})\left|G\left(q_{\text {phy }}^{2}\right)\right|^{2} .
$$

By comparing this expression with data on the $e^{+}+e^{-} \rightarrow p+\bar{p}$ total cross section, especially those data obtained by BABAR [12], which, thanks to the advantages of the ISR technique, practically start at the threshold, we get [17]

$$
\left|G\left(q_{\text {phy }}^{2}\right)\right|=1.00 \pm 0.05 .
$$

The fact that Sachs FFs have at the TL threshold $q_{\text {phy }}^{2}$ the same value owned at $q^{2}=0$, (even though in modulus ${ }^{5}$ ), appears quite inexplicable. Indeed, the unitary normalization at $q^{2}=0$ is a consequence of the charge and magnetization conservation which follows from the fact that FFs are, in this kinematical region, Fourier transforms of spatial distributions. Such an argument can not be invoked at TL $q^{2}$, simply because the interpretation of FFs in terms of Fourier transforms does not hold anymore. On the other hand, considering real FFs at $q_{\text {phy }}^{2}$ [18], the exact identity $G\left(q_{\text {phy }}^{2}\right)=1$ implies point-like behavior, in other words, proton and antiproton behave, at the production moment, like a pair of pointlike fermions [17].

- At large SL four-momenta, i.e., as $q^{2} \rightarrow-\infty$, pQCD and dimensional counting rule can be applied to gain information about the FF behavior, that is found to be well described by

${ }^{4}$ By using analytic continuation techniques, the derivative of $G_{E}\left(q^{2}\right)$ at SL $q^{2}$ can be expressed in terms of an integral of its TL modulus over the cut $\left(q_{\mathrm{th}}^{2}, \infty\right)$, in particular, with dispersion relations [1]

$$
r_{p}^{2}=\frac{6 \sqrt{q_{\mathrm{th}}^{2}}}{\pi} \int_{q_{\mathrm{th}}^{2}}^{\infty} \frac{\ln \left|G_{E}\left(q^{2}\right)\right|}{\left(q^{2}\right)^{2} \sqrt{q^{2}-q_{\mathrm{th}}^{2}}} d q^{2} .
$$

${ }^{5}$ Sachs FFs, at these values of $q^{2}$ are expected to be almost real [18]. 
power laws in $\left(q^{2}\right)^{-1}$. Moreover, by invoking the Phragmèn-Lindelöf theorem, FFs have to have, not only the same power-law, but rather the same limit, uniformly in all directions in the $q^{2}$ complex plane. This circumstance entails crucial consequences on what is expected at high $\left|q^{2}\right|$ in SL and TL regions, namely

$$
\lim _{q^{2} \rightarrow \infty} \frac{G_{E}\left(q^{2}\right)}{G_{E}\left(-q^{2}\right)}=\lim _{q^{2} \rightarrow \infty} \frac{G_{M}\left(q^{2}\right)}{G_{M}\left(-q^{2}\right)}=1
$$

where the numerators and the denominators contain the TL and SL limits respectively. As already discussed in Sec. 1, it is important to stress that TL FFs, which are complex, asymptotically become real.

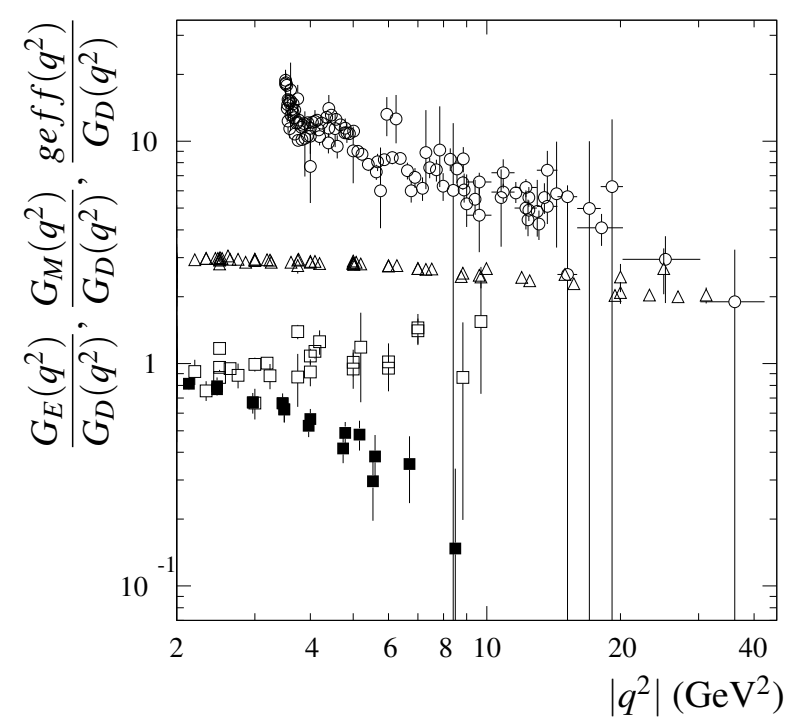

Figure 6: World data on proton FFs, in TL and SL regions, as function of $\left|q^{2}\right|$, scaled by dipole $G_{D}\left(q^{2}\right)$ (see text). From top to bottom: in the TL region effective FF (empty circles); magnetic FF in SL region (empty triangles); electric FF in SL region (empty squares) from unpolarized data and (solid squares) from experiments with polarization. Data and symbols are from Fig. 48 in Ref. [1].

Figure 6 shows the world data set on proton FFs, the data are reported as function of $\left|q^{2}\right|$ to compare TL and SL values, symmetric in $q^{2} \rightarrow-q^{2}$. To weaken the quick decreasing behavior of FFs, the data have been divided by the dipole $G_{D}\left(q^{2}\right)$, i.e.,

$$
G_{D}\left(q^{2}\right)=\frac{1}{\left[1-q^{2} /\left(0.71 \mathrm{GeV}^{2}\right)\right]^{2}} .
$$

The expected asymptotic coincidence seems to be fulfilled in case of the TL $G_{\text {eff }}\left(q^{2}\right)$, that at high squared momentum transferred tends to $\left|G_{M}\left(q^{2}\right)\right|$, see eq. (2.1), and the SL $G_{M}\left(q^{2}\right)$, represented in Fig. 6 by empty circles and empty triangles, respectively.

The situation becomes more difficult to interpret when the electric FF is considered. Firstly because it is not clear what value for $\left|G_{E}\left(q^{2}\right)\right|$, has to used in the TL region and secondly because in the SL region there are two different sets of data, obtained by means of Rosenbluth and polarization techniques represented, in Fig. 6, by solid and empty squares, respectively. 
It is interesting to notice that, assuming $\left|G_{E}\left(q^{2}\right)\right| \simeq\left|G_{M}\left(q^{2}\right)\right|$ in the TL region ${ }^{6}$, the SL values of $G_{E}\left(q^{2}\right)$ that seem to verify the asymptotic coincidence of eq. (3.1) are those obtained by means of the Rosenbluth technique, empty squares in Fig. 6, even though the polarization data, solid squares, are unanimously considered more reliable.

The asymptotic equality between a TL electric FF that coincides, in modulus, with $G_{M}\left(q^{2}\right)$, empty circles in Fig. 6, and its SL values obtained with the polarization technique, solid squares in Fig. 6, could be achieved, either by considering a drastic change of the $G_{E}\left(q^{2}\right)$ derivative that would reconcile its decreasing behavior with that of the dipole $G_{D}\left(q^{2}\right)$, or, and this is the most appealing possibility, by having a zero, so that $G_{E}\left(q^{2}\right)$ would become negative and $G_{E}\left(q^{2}\right) / G_{D}\left(q^{2}\right)$ would tend to -1 as $q^{2} \rightarrow-\infty$. In this case, as a consequence of the Phragmèn-Lindelöf theorem, also in the TL limit, $q^{2} \rightarrow \infty, G_{E}\left(q^{2}\right)$ would go to -1 . In Fig. 6 this behavior is masked since $G_{\text {eff }}\left(q^{2}\right)$ is the modulus of $G_{E}\left(q^{2}\right)$.

- Polarization phenomena play a peculiar role also in the TL region, especially when the annihilation $e^{+}+e^{-} \rightarrow p+\bar{p}$ is considered. Indeed, in this case the complexity of TL FFs "induces" polarization in the $p \bar{p}$ final state even though initial leptons are unpolarized. In particular, the component, perpendicular to the scattering plane, of the polarization vector of the outgoing proton is [19]

$$
P_{\perp}\left(q^{2}\right)=-\mu_{p} \frac{\sin (2 \theta)\left|R\left(q^{2}\right)\right|}{\sqrt{\tau}\left[1+\cos ^{2}(\theta)+\left|R\left(q^{2}\right)\right|^{2} \sin ^{2}(\theta) / \tau\right]} \sin \left[\rho\left(q^{2}\right)\right], \quad \tau=\frac{q^{2}}{4 M^{2}},
$$

where $\theta$ is the scattering angle, $R\left(q^{2}\right)$ is the ratio $R\left(q^{2}\right)=\mu_{p} G_{E}\left(q^{2}\right) / G_{M}\left(q^{2}\right)$, and $\rho\left(q^{2}\right)$ is its complex phase, i.e., $R\left(q^{2}\right)=\left|R\left(q^{2}\right)\right| e^{i \rho\left(q^{2}\right)}$. In other words, $\rho\left(q^{2}\right)$ represents the difference between the phases of the electric and magnetic FFs and, for real $q^{2}$ below the theoretical threshold $q_{\mathrm{th}}^{2}$, is a piece-wise constant function whose values are integer multiples of $\pi$ radians.

By assuming no zeros for $G_{M}\left(q^{2}\right)^{7}$, the function $R\left(q^{2}\right)$ is analytic in the same domain of the FFs and it is asymptotically constant, since $G_{E}\left(q^{2}\right)$ and $G_{M}\left(q^{2}\right)$ scale with the same power law. It follows that, by considering the generalized Lenvinson's theorem [20], the complex phase $\rho\left(q^{2}\right)$ has the limit (in radians)

$$
\lim _{q^{2} \rightarrow \infty} \rho\left(q^{2}\right)=N \pi
$$

where $N$ in the (integer) number of zeros that $R\left(q^{2}\right)$ has in the whole analyticity domain, i.e., the $q^{2}$ complex plane with the cut $\left(q_{\mathrm{th}}^{2}, \infty\right)$.

A measurement of such a complex phase, that could be accessed also through the single spin asymmetry in the reversed process $p^{\uparrow}+\bar{p} \rightarrow e^{+}+e^{-}$with polarized proton target $[1,19]$, could give unique information on the possible zero for $G_{E}\left(q^{2}\right)$ at SL $q^{2} \simeq-10 \mathrm{GeV}^{2}$, as the polarization data seem to suggest. Indeed, measuring values of $\rho\left(q^{2}\right)$ close to $\pi$ radians, at sufficiently high $q^{2}$ (few $\mathrm{GeV}^{2}$ ), would mean that $R\left(q^{2}\right)$ has a unique real zero at some $q^{2}<q_{\mathrm{th}}^{2}$. The possibility that such a zero is not real is excluded because the function $R\left(q^{2}\right)$,

\footnotetext{
${ }^{6}$ This assumption is supported by the BABAR data on the ratio $\left|G_{E}\left(q^{2}\right) / G_{M}\left(q^{2}\right)\right|$ shown in Fig. 4 .
}

${ }^{7}$ This assumption is supported by the result of Ref. [18]. 
being analytic and real in the portion of real axis contained in its domain, fulfills the Schwarz reflection principle ${ }^{8}$ so that, not real zeros occur only in pairs of complex conjugates.

\section{A strategy rather than several tactics}

Rather than venture into a close examination or, even only, a roundup of all the models developed to describe hadron FFs, we would prefer to set the "boundary conditions" that should be fulfilled by any reliable model, that pretends to describe FFs in the whole kinematical region (as it should be), and to make some example.

Unfortunately, the investigation of FFs is particularly intriguing in the low- $\left|q^{2}\right|$ kinematical region, where QCD is non-perturbative and hence is useless to compute directly FFs by describing the nucleons as three-quark bound states. On the other hand, when $\left|q^{2}\right|$ increases, as already discussed, pQCD gives predictions, mainly concerning the asymptotic behavior. Nevertheless, it remains a task of the experiments, the "burden" of establishing whether $\left|q^{2}\right|$ is large enough to be considered asymptotic.

Even though FFs can not be computed in the framework of a QFT, first-principle considerations can be used to define those general features, described in the previous sections, that should be embodied by any reliable model.

The function $\mathscr{F}\left(q^{2}\right)$ that describes a Sachs FF should have the following properties:

- it must be analytic for all complex value of $q^{2} \notin\left(q_{\mathrm{th}}^{2}, \infty\right)$;

- it must be real for real $q^{2}$, with $q^{2} \leq q_{\mathrm{th}}^{2}$;

- it must be complex over the TL cut, i.e., for $q^{2}=q^{2^{\prime}}+i \varepsilon$, with $\varepsilon \rightarrow 0^{+}$and $q^{2^{\prime}}>q_{\mathrm{th}}^{2}$;

- it must fulfill the Schwarz reflection principle;

- it must behave as $\mathscr{F}\left(q^{2}\right)=O\left[\left(q^{2}\right)^{-2}\right]$, for $q^{2} \rightarrow \pm \infty$.

In addition there are the conditions on both Sachs FFs:

•• the coincidence at the physical threshold: $G_{E}\left(q_{\text {phy }}^{2}\right)=G_{M}\left(q_{\text {phy }}^{2}\right)$;

-. the asymptotic power law: $\lim _{\left|q^{2}\right| \rightarrow \infty} \frac{G_{E}\left(q^{2}\right)}{G_{M}\left(q^{2}\right)}=R_{\infty}$, where $R_{\infty}$ is a constant (in any direction in the $q^{2}$ complex plane and, in particular, in the SL and TL ones).

This list defines the key points of our strategy to attack the problem of building up a reliable description of nucleon FFs. On the other hand, models conceived for a particular kinematical region that, in many cases, give non-analytic functions for FFs, can be seen as good tactics that could allow to "win a battle", but, missing the first-principles properties, "not the war".

\footnotetext{
${ }^{8}$ See footnote 2 .
} 


\subsection{A historical and still fundamental model}

Vector meson dominance (VMD) is a phenomenological tool that allows to describe the electromagnetic interactions of hadrons by means of a "QFT language". Indeed, diagrams à la Feynman are used to parametrize, order by order, the coupling of a physical hadron (external line), $h$, with real or virtual photons. The only prescription is that, in any elementary vertex $h \gamma^{(*)} h$, the photon line is not attached directly to the hadronic one but it is mediated by all the vector mesons carrying the same quantum numbers of the photon, namely with $J^{P C}=1^{--}$.

It is just for this close relationship with a standard QFT that the amplitudes computed in the framework of VMD have the same properties of those of QED, in particular, they are obtained as well defined functions of $q^{2}$ and hence can be analytically continued from one kinematical region to another.

In addition, by using energy-dependent and relativistic propagators for the intermediate vector mesons (see e.g. Ref. [21]), all the required analytic features of FFs, that are the amplitudes of the elementary vertex $h \gamma^{*} h$, as the discontinuity cut and the asymptotic power-law, can be reproduced. The success of models based on VMD lies in their capacity of reproducing the data of all nucleon FFs in all kinematical regions with a minimal number of parameters, all of them having a well established physical meaning (masses, widths, couplings).

It appears quite intriguing to notice how, other descriptions of nucleon FFs, achieved by means of different theoretical tools (chiral perturbation theory, conformal field theory, ...), but all with the aim of being extensible to the whole kinematical region, arrive to functional forms which reproduce the sum of vector meson propagators (resonances) typical of VMD.

\section{Conclusion}

Despite nucleons are the primary test bed of particles physics and despite several decades of experimental investigations and theoretical studies, not few aspects of FFs are still obscure.

The TL region, less explored than its SL counterpart, appears as the "treasure island". Many experimental groups are working on that in a well organized complementary scenario.

In particular, the BESIII experiment at the BEPCII $e^{+} e^{-}$-collider [22] will measure the ratio $\left|G_{E}\left(q^{2}\right) / G_{M}\left(q^{2}\right)\right|$ at a few-percent level in the CM energy range $\sqrt{q_{\text {phy }}^{2}} \leq \sqrt{q^{2}} \leq 3 \mathrm{GeV}$.

The experiments CMD-3 and CND at the VEPP-2000 $e^{+} e^{-}$-collider [23] will explore the protonantiproton, as well as the neutron-antineutron threshold region by trying to verify the identity $G_{E}\left(q_{\text {phy }}^{2}\right)=G_{M}\left(q_{\text {phy }}^{2}\right)$ and hence the engaging possibility of having a singularity for the Dirac and Pauli FFs, at the physical threshold [1].

The future PANDA experiment at the FAIR facility [24] will extract individual moduli of Sachs FFs at high momentum transfer in the reversed channel $p+\bar{p} \rightarrow e^{+}+e^{-}$.

On the other hand, the theory community works in developing new phenomenological concepts, ideas and also computational tools, that, with the help of the forthcoming data, would answer many, still open, questions. 


\section{References}

[1] S. Pacetti, R. Baldini Ferroli and E. Tomasi-Gustafsson, Phys. Rept. 550-551 (2014) 1.

[2] C. Adamuscin, E. A. Kuraev, E. Tomasi-Gustafsson, F. Maas, Phys. Rev. C 75 (2004) 045205.

[3] V. Matveev, R. Muradyan, A. Tavkhelidze, Teor. Mat. Fiz. 15 (1973) 332;

S. J. Brodsky, G.R. Farrar, Phys. Rev. Lett. 31 (1973) 1153.

[4] E. Titchmarsh, The Theory of Functions, Oxford science publications, Oxford University Press, 1939.

[5] R. Hofstadter, F. Bumiller, M. Croissiaux, Phys. Rev. Lett. 5 (1960) 263.

[6] M. Rosenbluth, Phys. Rev. 79 (1950) 615.

[7] A. Akhiezer, M. Rekalo, Sov. Phys. Dokl. 13 (1968) 572. A. Akhiezer, M. Rekalo, Sov. J. Part. Nucl. 4 (1974) 277.

[8] M. Jones, et al., Phys. Rev. Lett. 84 (2000) 1398.

[9] A. Zichichi, S. Berman, N. Cabibbo, R. Gatto, Nuovo Cim. 24 (1962) 170.

[10] G. Bardin, et al., Nucl. Phys. B 411 (1994) 3.

[11] B. Aubert et al. (BABAR Collaboration), Phys. Rev. D 73 (2006) 012005.

[12] J. Lees, et al. (BABAR Collaboration), Phys. Rev. D 87 (2013) 092005.

[13] A. V. Afanasev, S. J. Brodsky, C. E. Carlson, Y. C. Chen and M. Vanderhaeghen, Phys. Rev. D 72 (2005) 013008.

[14] E. A. Kuraev, A. I. Ahmadov, Y. M. Bystritskiy and E. Tomasi-Gustafsson, Phys. Rev. C 89 (2014) 065207.

[15] R. Pohl, A. Antognini, F. Nez, F. D. Amaro, F. Biraben, J. M. R. Cardoso, D. S. Covita and A. Dax et al., Nature 466 (2010) 213;

A. Antognini, F. Nez, K. Schuhmann, F. D. Amaro, FrancoisBiraben, J. M. R. Cardoso, D. S. Covita and A. Dax et al., Science 339 (2013) 417.

[16] A. Sakharov, Pisma Zh. Eksp. Teor. Fiz. 5 (1967) 32;

A. Sommerfeld, Atombau und Spektralliniem, V Edition, Vol. 2, Vieweg, Braunschweig, 1944.

[17] R. Baldini, S. Pacetti, A. Zallo, A. Zichichi, Eur. Phys. J. A 39 (2009) 315.

[18] R. Baldini, S. Dubnicka, P. Gauzzi, S. Pacetti, E. Pasqualucci and Y. Srivastava, Eur. Phys. J. C 11 (1999) 709.

[19] A. Z. Dubnickova, S. Dubnicka and M. P. Rekalo, Nuovo Cim. A 109 (1996) 241.

[20] N. Levinson, Danske Vid. Selsk. Math. Fys. Medd. 25 (1949) 1.

[21] E. L. Lomon and S. Pacetti, Phys. Rev. D 85 (2012) 113004 [Erratum-ibid. D 86 (2012) 039901].

[22] The BESIII experiment, http://bes3.ihep.ac.cn/.

[23] The CMD-3 experiment, http://cmd.inp.nsk.su/. The CND experiment, http://wwwsnd.inp.nsk.su/.

[24] The PANDA experiment, http://www-panda.gsi.de/. 